\title{
Memory without content? Radical enactivism and (post)causal theories of memory* $^{*}$
}

\author{
Kourken Michaelian ${ }^{\dagger} \quad$ André Sant'Anna
}

\begin{abstract}
Radical enactivism, an increasingly influential approach to cognition in general, has recently been applied to memory in particular, with Hutto and Peeters (2018) providing the first systematic discussion of the implications of the approach for mainstream philosophical theories of memory. Hutto and Peeters argue that radical enactivism, which entails a conception of memory traces as contentless, is fundamentally at odds with current causal and postcausal theories, which remain committed to a conception of traces as contentful: on their view, if radical enactivism is right, then the relevant theories are wrong. Partisans of the theories in question might respond to Hutto and Peeters' argument in two ways. First, they might challenge radical enactivism itself. Second, they might challenge the conditional claim that, if radical enactivism is right, then their theories are wrong. In this paper, we develop the latter response, arguing that, appearances to the contrary notwithstanding, radical enactivism in fact aligns neatly with an emerging tendency in the philosophy of memory: radical enactivists and causal and postcausal theorists of memory have begun to converge, for distinct but compatible reasons, on a contentless conception of memory traces.
\end{abstract}

\section{Radical enactivism meets the philosophy of memory}

In opposition to the representationalist approaches that have long dominated cognitive science, Hutto and Myin's $(2013 ; 2017)$ radical enactivism maintains that, as a general principle, cognition is best explained not in terms of representation but rather in terms of the reactivation, in response to current environmental stimuli, of neural patterns shaped by the subject's previous interactions with his environment. Critics of this alternative approach have charged that it is unable to explain paradigmatically representational forms of cognition, including remembering. Earlier work on radical enactivism (2013) did indeed focus primarily on basic forms of cognition, such as perception, but radical enactivists

\footnotetext{
*Thanks for feedback to audiences at the Naturally Evolving Minds conference at the University of Wollongong in February 2018 and the Memory and Perception: Fishing for Connections workshop at the University of Otago in May 2018. Thanks also for written comments to Carl Craver and John Sutton and for extremely interesting reports to two referees.

${ }^{\dagger}$ kourken.michaelian@univ-grenoble-alpes.fr
} 
have recently turned their attention to remembering and other non-basic forms of cognition (Hutto and Myin, 2017). In the first systematic discussion of the relationship between radical enactivism and mainstream philosophical theories of memory, Hutto and Peeters (2018) argue that the account that emerges when radical enactivism is applied to memory poses a significant challenge to current causal and postcausal theories.

This paper aims to get beyond Hutto and Peeters' "radical" rhetoric in order to draw out the implications of their account for a cluster of (post)causal theories: the classical causal theory (Martin and Deutscher, 1966) and a neoclassical variant thereof (Bernecker, 2010), the simulation theory (Michaelian, 2016b), and the recently-proposed procedural causal theory (Perrin, 2018). ${ }^{1}$ There are, of course, other theories on which we might have focused, but this particular cluster constitutes a natural starting point. The (neo)classical causal theory continues to occupy a dominant position in the philosophy of memory and is one of Hutto and Peeters' primary targets. The simulation theory is a descendant of and opponent to the causal theory and, despite being motivated by many of the same considerations that motivate the radical enactivist account of remembering, is singled out by Hutto and Peeters for special criticism: on their view, the simulation theory, though it differs from the causal theory in important respects, retains those features of the latter that render it incompatible with radical enactivism. The procedural causal theory, while it is not discussed by Hutto and Peeters, is likewise motivated by considerations similar to those that motivate radical enactivism; it promises, moreover, to point the way to a version of the simulation theory that jettisons the features singled out for criticism by Hutto and Peeters.

We proceed as follows. In section 2 , we zero in on the "no content" claimaccording to which memory traces should be understood as not having contentthat we see as constituting the core of Hutto and Peeters' account. In section 3 , we reconstruct their argument for the claim as it pertains to procedural, semantic, and episodic memory, and, in section 4, we explore its implications for mainstream theories of episodic remembering, concluding that Hutto and Peeters may well be right about the contentless character of traces but that they are nevertheless wrong about the compatibility of radical enactivism and certain of these theories: recent causal theories (namely, the procedural causal theory) and postcausal theories (namely, a "nontransmissionist" version of the simulation theory) may not be motivated by the sort of general hostility to mental content that animates radical enactivism, but they can in fact take the "no content" claim on board. Section 5 brings the paper to a close with a discussion of the implications of this conclusion for ongoing debates over the taxonomy of memory and memory errors.

Before proceeding, we pause to emphasize two points. First, nothing in what follows is meant to rule out the possibility that radical enactivism will

\footnotetext{
${ }^{1}$ Our focus here will be exclusively on radical enactivism, and we will have nothing to say about the implications of other forms of enactivism for the philosophy of memory. Hutto and Myin (2013) argue that "sensorimotor enactivism" (Hurley, 1998; O'Regan and Noë, 2001; Noë, 2004) takes perception to be a matter of embodied action but does not entirely give up on the idea that perception is representational, while "autopoeitic enactivism" (Varela et al., 1991; Thompson, 2007) relies on metaphors (e.g., "interpretation", "sense-making") that are often understood in representational terms. Radical enactivism, we take it, is meant to differ from these other enactivist approaches in that it attempts to break entirely with the view that cognition involves representation.
} 
ultimately turn out to be incompatible with the procedural causal theory or the simulation theory due to disagreement over issues other than the contentless or contentful character of memory traces. Where potential tensions between these theories and radical enactivism are evident, we note them, but our aim here is the restricted one of determining whether (post)causal theorists can take the core of Hutto and Peeters' account - that is, the "no content" claim - on board, and we remain agnostic with respect to the implications of radical enactivism as a whole. Second, we likewise remain agnostic with respect to the ultimate viability of radical enactivism in its own right, taken either as an approach to memory in particular or as an approach to cognition in general. ${ }^{2}$ The perspective that we adopt here is that of the philosophy of memory, in the sense that we are concerned specifically with the relationship between radical enactivism and philosophical theories of memory - our aim is not to assess the success of Hutto and Peeters' argument for radical enactivism about memory or that of the more general radical enactivist arguments advanced by Hutto, Myin, and others.

\section{Content, construction, and cognitive exten- sion}

In developing their account, Hutto and Peeters appeal to three distinct bodies of theory, invoking treatments of remembering as, first, extended and embodied (Clark and Chalmers, 1998; Sutton and Williamson, 2014), second, reconstructive, simulative, or imaginative (Perrin and Michaelian, 2017; Sant'Anna, 2018; Michaelian et al., 201x), and, third, nonrepresentational or contentless (Loader, 2013; De Brigard, 2014). ${ }^{3}$ The aspects of remembering on which the first two of these focus would seem, on the face of it, to pair naturally with an account of remembering as involving the retrieval of stored content, but Hutto and Peeters argue that they are in fact best accommodated by an enactivist version of the sort of contentless account suggested by the third. An enactivist account of remembering as "creative, dynamic, and wide-reaching", they argue, "can more easily accommodate [the relevant empirical findings] than cognitivist accounts that conceive of remembering as always involving passive recollections that occur wholly and solely inside heads" $(2018,98)$. They continue: "[a]ccording to our enactivist proposal, the roots of remembering can be understood in line with [the principle that] invoking neural, bodily and environmental factors can all make equally important contributions when it comes to explaining and characterizing cognitive activity" (2018, 98). The relevant bodies of theory are discussed as

\footnotetext{
${ }^{2}$ Radical enactivism has been the target of a number of objections (see Myin and Hutto 2015). Some have objected that, given the entrenched status of the concept of content in philosophy and its explanatory utility in cognitive science, radical enactivists have not provided sufficient reason for abandoning it (Campbell, 2014; Shapiro, 2014; Colombo, 2014). Others have objected that radical enactivists have not adequately taken concepts of representation that do not appeal to accuracy conditions into account (Roberts, 2013; Gładziejewski, 2015). And others have objected that radical enactivists have not provided clear positive accounts of specific forms of cognition, as opposed to negative arguments against representationalist accounts (Shapiro, 2014). The account of remembering proposed by Hutto and Peeters can be seen as providing a partial response to the last of these objections.

${ }^{3}$ Hutto and Peeters read De Brigard as endorsing a contentless conception of traces. Though he does not explicitly make this claim, we will not challenge their reading here.
} 
appropriate in sections $3-4$ below. ${ }^{4}$ The point to note at this stage is just that the account that we will refer to as "radical enactivism about memory" foregrounds, first, the role of environmental (and bodily) resources in remembering, and second, the reconstructive character of remembering.

In Hutto and Peeters' view, these first two features of the account already mean that radical enactivism about memory is at odds with mainstream theories of memory. We will see, however, that many mainstream theories can and do acknowledge that remembering is both extended and reconstructive. Its emphasis on the extended and reconstructive aspects of remembering, then, is not what is distinctive of radical enactivism about memory. What is distinctive - or what at least initially appears to be distinctive - is a third feature of the account, namely, its stance with respect to content. Hutto and Peeters argue that "we achieve a stronger and more elegant account of the basis of remembering by going radically enactivist-viz. by abandoning the widely held assumption that remembering always and everywhere involves the retrieval of stored information or content in order to represent events" (2018, 98; emphasis added). The gist of their claim is clear enough, but it is worth pointing out that the way they formulate it here is potentially misleading. Radical enactivism about memory does not (as their subsequent discussion makes clear) simply reject the claim that remembering always involves the retrieval of stored content, which would not rule out the possibility that remembering sometimes involves the retrieval of stored content; instead, it endorses the claim that remembering never involves the retrieval of stored content. We will refer to this as the "no content" claim $(\mathrm{NC}) .^{5}$

$\mathrm{NC}$ is, as stated, ambiguous, in the sense that it might be taken to apply either to memory in general or, instead, to one or more specific kinds of memory. It is standard, both in philosophy and in the empirical sciences of memory, to distinguish between procedural memory (roughly, "remembering how") and declarative memory ("remembering that") (Werning and Cheng, 2017). Within the category of declarative memory, in turn, it is standard to distinguish between episodic memory and semantic memory. Episodic memory is memory for experienced past events (e.g., remembering visiting Wellington for the first time) (Tulving, 1972) and is sometimes thought to essentially involve a specific form of consciousness, autonoesis (Tulving 2005; Klein 2015; see section 5 below). Semantic memory, on the other hand, is memory for facts in general and need not refer to specific events (e.g., remembering that Wellington is the capital of New Zealand).

Depending on the kind or kinds of memory to which NC is applied, we end up with a more or less surprising or "radical" approach:

(NC-procedural) Procedural remembering does not involve the retrieval of stored content.

(NC-semantic) Semantic remembering does not involve the retrieval of stored content.

\footnotetext{
${ }^{4}$ With respect to the first body of theory, we focus on extended rather than embodied aspects of remembering. This is in keeping with Hutto and Peeters' own focus.

${ }^{5}$ Since our focus here is on radical enactivism, we will have nothing to say about the relationship between Hutto and Peeters' argument for NC and historical attacks on contentful traces. For background on this history, see chapter 16 of Sutton 1998.
} 
(NC-episodic) Episodic remembering does not involve the retrieval of stored content.

While there is no obvious reason for adopting NC-semantic and NC-episodic without also adopting NC-procedural, we will see that one might well want to adopt NC-procedural without also adopting NC-semantic or NC-episodic. We will also see that NC-procedural is not particularly surprising. In contrast, NC-semantic is already highly counterintuitive, and NC-episodic is yet more counterintuitive: it is (to put it mildly) difficult to see how one might hope to provide an account of episodic memory - which is increasingly understood as a form of episodic imagination, which is, in turn, usually taken to be a paradigm representational capacity - without invoking informational or representational content.

\section{The "no content" claim}

One might thus expect Hutto and Peeters to begin straightaway with treatments of episodic remembering as a reconstructive, simulative, or imaginative process, but they begin, instead, with Clark and Chalmers' (1998) treatment of remembering as a form of extended cognition, invoking the now-familiar thought experiment of Otto and his notebook and Inga and her brain, the result of which is supposed to be that Otto's notebook, said to be his "external memory", is just as much a part of his mind as Inga's "internal memory" is of hers. It is worth following them in this, as doing so will serve to clarify what, exactly, is at stake in NC.

When they introduced their thought experiment, Clark and Chalmers described the relationship between Otto and his notebook in thoroughly representationalist terms, taking the latter to be a store, functionally equivalent to Inga's memory, for information or content: just as Inga (they suppose) forms beliefs by retrieving content from her internal memory, Otto is said to form beliefs by retrieving content from his external memory. If this description of the case were taken for granted, cognitive extension would appear to be straightforwardly incompatible with $\mathrm{NC}$ and hence with radical enactivism about memory. Hutto and Peeters, however, reject Clark and Chalmers" "first-wave" (Sutton, 2010) take on cognitive extension, following Loader (2013) in arguing that it rests on an untenable "commodity conception" of memories.

The commodity conception treats engrams (internal memory traces) and exograms (external memory traces) as being effectively interchangeable - units of content capable, in principle, of being stored in brains, notebooks, and various other locations. Now, philosophers of memory have often observed that the storage format employed by internal memory in fact differs greatly from that employed by external memory (Sutton, 2010; Michaelian, 2012), and, from the perspective of the philosophy of memory, we thus might initially suspect that Hutto and Peeters reject the first-wave take on cognitive extension because they reject its assumption that internal and external memory share a common storage format. The suspicion would, however, be misplaced.

Clark and Chalmers' argument notoriously appeals to the notion of parity between internal and external resources, the idea being that, if an external resource performs a role that would be counted as cognitive were it performed by an internal resource, then the external resource is just as much a part of the 
relevant cognitive system as the internal resource would be. Later arguments for the extended cognition hypothesis, in contrast, appeal to the notion of complementarity between internal and external resources, with Sutton (2010), for example, maintaining that the distinct roles played by internal and external resources are precisely what make the hypothesis plausible: the differences with respect to storage format between engrams and exograms, in particular, enable external memory to play roles that internal memory is ill-suited to play, thus enabling subjects to perform cognitive tasks that they would otherwise be unable to perform, and satisfactory explanations of our cognitive performance will therefore often treat cognition as extended.

Sutton builds here on his own earlier work (Sutton, 1998) on the distributed character of engrams. This contrasts sharply with the local character of exograms: whereas external memory traces (such as the entries in Otto's notebook) are typically discrete, stable, nonoverlapping entities carrying explicit content, information storage in internal memory is, in Sutton's view, blended, malleable, superpositional, and implicit, roughly in the manner of connectionist networks. ${ }^{6}$ Targeting Sutton in particular, Hutto and Peeters reject this "second-wave" extended cognition just as resoundingly as they reject first-wave extended cognition, charging that

[d] espite acknowledging that the putative informational contents of our memories are stored in quite different ways in biological memory, as opposed to external devices, second wave extended mind theorists remain committed to [the contrary of NC]. Thus, they remain committed to a content-based view of remembering to the extent that they assume that there are contents to be stored at all-viz. to the extent that they retain the idea that remembering essentially involves receiving, encoding, and retrieving stored contentful information about particular events or episodes. (2018, 103)

It thus becomes evident that their concern is not with storage format but rather with the idea of storage as such: on their view, content is stored neither in Otto's notebook nor in Inga's brain, regardless of whether these share a common format.

Given the kinds of contents putatively stored in Otto's notebook and the role that these are said to play in his cognitive life, Clark and Chalmers' thought experiment, to the extent that it is about memory, concerns semantic memory in particular. Hutto and Peeters' discussion, however, makes clear that they mean to endorse not just NC-semantic but also NC-episodic and NC-procedural: regardless of the kind of memory at issue, in other words, memory traces are not contentful. ${ }^{7}$ Having determined that what is at stake in $\mathrm{NC}$ is the very idea of stored content, we can set the question of the extended character of remembering aside (though we will need to briefly come back to extended cognition below). If we endorse $\mathrm{NC}$, then we can (if Hutto and Peeters are right) endorse the

\footnotetext{
${ }^{6}$ See Robins 2016 b for detailed discussion of the relationship between connectionist networks and networks of the kind thought to be involved in memory.

${ }^{7}$ In order to avoid terminological confusion, we note that, while Hutto and Peeters (like Perrin 2018; see section 4) would prefer not to refer to memory traces at all, this is because they take it a contentful conception of traces for granted; they would presumably be willing to refer to traces on the condition that these are understood in line with the contentless conception outlined below.
} 
extended cognition hypothesis, adopting a version of the hypothesis on which neither internal nor external memory deals in stored content. If we reject $\mathrm{NC}$, then we can (pace Adams and Aizawa 2008 and others who have posited a difference between "intrinsic" internal content and "derived" external content) likewise endorse the extended cognition hypothesis, adopting a version of the hypothesis on which both internal and external memory deal in stored content. Extended cognition, in other words, does not drive a wedge between radical enactivism about memory and mainstream theories of memory-mainstream theories are compatible with the first key feature of radical enactivism about memory.

The motivations for the remaining two key features of the account - its emphasis on the reconstructive character of remembering, and NC itself - are intertwined with the distributed conception of (internal) memory traces introduced above. Part of Hutto and Peeters' motivation for rejecting a conception of memory traces as contentful stems from their commitment to the broader radical enactivist project of accounting for as much of cognition as possible without reference to content. ${ }^{8}$ But their rejection of the contentful conception of traces is also motivated more specifically by their perception of the greater faithfulness of a contentless conception to the relevant science. Thus they approvingly cite De Brigard's take on the empirical literature: ${ }^{9}$

"Storing" is a rather misleading term. What seems to occur when we encode information is the strengthening of neural connections due to the co-activation of different regions of the brain [...]. A memory trace is the dispositional property these regions have to re-activate, when triggered by the right cue, in roughly the same pattern of activation they underwent during encoding. (2014, 169)

We may, if De Brigard (as interpreted by Hutto and Peeters) is right, continue to refer to "memory traces", on the condition that these are not understood as being "commodities" - items that might in principle be encoded, stored and retrieved. Traces are, instead, to be understood as being dispositions of a larger network. While it may be useful to retain the vocabulary of encoding, storage, and retrieval in order to have distinct labels for distinct stages of the memory process, remembering, strictly speaking, does not involve the storage of content. "Encoding" refers, instead, to the creation of a disposition, with "retrieval" referring to the activation of the disposition and "storage" referring to the period in between encoding and retrieval, during which the disposition is inactive. ${ }^{10}$

\footnotetext{
${ }^{8}$ This project is motivated, in turn, by the claims, first, that there is no naturalistically respectable account of content available (the so-called "hard problem of content") and, second, that eliminating reference to content in cognitive science does not result in loss of explanatory power (Hutto and Myin, 2013, 2017).

${ }^{9}$ The literature in question is voluminous, and we will not attempt to duplicate De Brigard's summary and interpretation of it here.

${ }^{10}$ One might object here that a disposition to produce a certain content is naturally viewed as carrying that content. While it is not our aim here to defend Hutto and Peeters' move from the distributed conception to the contentless conception, we note that the obvious response to this objection is to point out that the issue is about storage of content and that a disposition is simply not the sort of entity that might store content. One might further object that the notion of a contentless trace is unintuitive and that a much more detailed statement of the contentless conception is required before radical enactivism about memory can be properly assessed. While our project here, which is concerned with the relationships among existing theories rather than with developing those theories in further detail, does not require
} 
There is a clear echo here of Sutton's distributed conception of traces, which similarly understands traces in terms of modifications of the strengths of connections among nodes in a network. The overall relationship between the distributed conception and the contentless conception is not, however, immediately clear, as Sutton treats traces as having (implicit) content. We come back to this relationship below. The point to note, for the moment, is that the contentless conception, on which, regardless of whether they originate in contentful states (at encoding) or result in contentful states (at retrieval), traces are not themselves contentful, is meant to respect what the sciences of memory have revealed about the workings of the memory process.

\subsection{Procedural memory without content}

De Brigard's argument for the contentless conception is persuasive - and we will accordingly refer to "encoding", "storage", and "retrieval" without presuming that content is literally encoded, stored, and retrieved-but it is not immediately obvious whether, if we adopt that conception, we can account for all forms of remembering. Hutto and Peeters' argument to the effect that we can gets under way with an attempt to establish NC-procedural. This portion of their argument is relatively straightforward, and we will not spend any time reviewing it here. "Radical" rhetoric notwithstanding, NC-procedural is not a particularly surprising claim: there is little temptation to suppose that procedural remembering involves the retrieval of stored content, simply because the outputs of procedural remembering (actions of various sorts) do not themselves appear to be contentful, and most philosophers of memory thus arguably endorse the claim.

Michaelian's (2016b) simulation theory of episodic memory, for example, is singled out by Hutto and Peeters as being incompatible with radical enactivism, but Michaelian himself argues that the noninvolvement of stored content in procedural remembering marks a difference in kind, within the broad category of memory, between (contentless) procedural memory and (contentful) declarative memory. There are important differences between the simulation theory and the causal theory, but none that would prevent a causal theorist from running the same argument. This argument would, of course, appear to be incompatible with NC-semantic and NC-episodic. We argue in section 5 that the argument can easily be reworked so as to remove any incompatibility, but what matters, at this stage of the dialectic, is that there is no reason to suspect any incompatibility between radical enactivism about memory and mainstream philosophical theories of memory with respect to procedural memory: while there are occasional references to "procedural information" in the psychological literature (see Michaelian 2016b), mainstream philosophical theories of memory can adopt NC-procedural. The question of the compatibility of radical enactivism about memory and mainstream theories of memory thus turns on NC-semantic and NC-episodic.

us to undertake this, we acknowledge that radical enactivists (and procedural causal and nontransmissionist simulation theorists; see section 4) will eventually need to develop the contentless conception in more detail. 


\subsection{Semantic memory without content}

In order to argue for NC-semantic, Hutto and Peeters turn back to Clark and Chalmers' thought experiment. Otto, in their view, forms a contentful semantic memory despite the fact that he retrieves no content from his external memory. Inga, they suggest, likewise forms a contentful semantic memory despite the fact that she retrieves no content from her internal memory. It is not entirely clear how the transition from Otto's contentless external memory "store" to his contentful "retrieved" memory is supposed to be made, but this need not concern us here. The transition from Inga's contentless internal memory store to her contentful retrieved memory is, it appears, supposed to be made in line with De Brigard's story: We begin with the coactivation of brain regions, underwriting a contentful state. This leads (through the process of encoding) to the creation of a disposition (storage). When activated by an appropriate cue, the disposition leads to the coactivation of brain regions similar to the previous coactivation, underwriting a contentful state similar to previous state (retrieval). This story raises a pair of worries. First, assuming that semantic thoughts and memories are indeed contentful states, it is not clear what process is supposed to take us from a contentful state (the initial semantic thought) to a contentless state (the encoded memory) and back to a contentful state (the retrieved memory). Second, it is not in fact clear whether semantic memories are meant to be understood as being contentful in their own right or whether they are instead meant to be understood as acquiring content only when combined with some further factor. In the case of episodic memory, as we will see, the suggestion seems to be that the products of retrieval are not contentful in their own right but acquire content only when the subject is in possession of certain sociocultural practices (Hutto and Peeters 2018, 110; see also Hutto and Myin 2017, ch. 9 and Hutto 2017). It would thus not be surprising if Hutto and Peeter's view entailed that the products of semantic retrieval are not themselves contentful but acquire content only in certain sociocultural contexts.

While this aspect of their view is not entirely clear, we set it aside for the moment, as we come back to NC-semantic in section 5. The important point, for present purposes, is that, regardless of the details, Hutto and Peeters seem to accept the claim that in semantic memory we ultimately arrive at a contenful state. Thus, rather than offering an explicit assessment of Hutto and Peeters' account of semantic remembering at this stage, we will turn directly to their account of episodic remembering. It would, given that semantic memory and episodic memory are both forms of declarative memory, be surprising if NCsemantic were true but not NC-episodic or vice versa - it is difficult to imagine a coherent overall account on which episodic remembering does not involve the retrieval of stored content while semantic remembering does or on which semantic remembering does not involve the retrieval of stored content while episodic remembering does. Admittedly, the precise relationship between episodic memory and semantic memory is not entirely clear. On some views, there may be a single declarative memory system responsible for both episodic and semantic memory. Klein (2015), for example, has argued that episodic and semantic memory both involve retrieval of the same sort of content and are distinguished primarily by their respective phenomenologies. On other views, episodic and semantic memory may amount to distinct systems. But even on views of the latter sort, episodic and semantic memory interact in a direct manner. Auto- 
biographical memory, for example, does not, on most accounts, correspond to a distinct system but is rather a complex capacity requiring the integration of episodic memories of specific events with more general semantic memories of life stages. Given a view of either sort, then, it would appear not to be workable to conjoin a contentless view of one kind of memory with a contentful view of the other.

Anticipating our discussion of episodic memory below, one might worry that, while it is relatively natural to view episodic memory as a form of imagination, it is much less natural to view semantic memory as a form of imagination and that, in view of this difference between the two forms of memory, it would not be surprising were there to turn out to be a difference with respect to storage of content between episodic and semantic memory. In response to this worry, we point out that, while it may indeed be less natural to view semantic memory as a form of imagination, semantic remembering may nevertheless turn out to have an imaginative character. Michaelian (2018), for example, builds on views (e.g., Goldman 2006; see Arcangeli 201x for discussion) on which both semantic imagination (also referred to as suppositional imagination) and episodic imagination (also referred to as enactment imagination) are both a matter of simulating mental states to argue that it may ultimately be possible to extend the simulation theory from episodic to semantic memory. Klein (2015), too, though he singles episodic memory out as having a special status (see section 5), posits a fundamental similarity between episodic and semantic memory, arguing that the difference between them is purely phenomenological; this similarly suggests that, if we are entitled to describe episodic memory as a form of imagination, we are likewise entitled to describe semantic memory as a form of imagination. Of course, the availability of these views does not settle the matter, and one might reinforce the worry by noting that, while the relationship between episodic memory and imagination has been established by research on episodic memory as a form of mental time travel tightly linked episodic future thought (Perrin and Michaelian, 2017; Sant'Anna, 2018), no similar link between semantic memory and an analogous future-oriented capacity has been established. In response, we point out that, while most research on memory as a form of mental time travel so far has focused on episodic memory and episodic future thought, there is increasing emphasis on the role of semantic memory in future-oriented mental time travel (Irish, 2016) and indeed on properly semantic forms of futureoriented thought (Klein et al., 2002; Szpunar et al., 2016). These considerations do not conclusively establish that episodic memory and semantic memory do not differ significantly with respect to their relationship to imagination, but they do suggest that it is reasonable to suppose that $\mathrm{NC}$-episodic and NC-semantic will stand or fall together.

\subsection{Episodic memory without content}

Turning to NC-episodic, Hutto and Peeters emphasize that, in line with empirical research on memory as a form of mental time travel, recent philosophical theories treat episodic remembering as a thoroughly reconstructive process, a matter of imagining or simulating past events. Such theories, they note, might appear to pose a problem for radical enactivism about memory: "It might be thought that if episodic memory just is, or centrally involves, simulative imagining then it must be representational. [...] [I]magining, by its very nature, 
deals with presence in absence. If all imagining is necessarily representational then defeat for radically enactive accounts of imagination and memory follows briskly" (2018, 110-111). The basic thought here is straightforward-if radical enactivism is committed to the claim that retrieved episodic memories do not have representational content, while an understanding of episodic memory as a form of imagination is bound to imply that retrieved episodic memories do have representational content, then radical enactivism cannot accommodate such an understanding of episodic memory - but it is nonetheless puzzling that Hutto and Peeters take this to be the decisive point, for their argument otherwise focuses on the presence of content during storage, not after retrieval: NC-episodic, as we have interpreted it, denies that episodic remembering involves the retrieval of stored content, since it denies that traces are contentful, but - bearing in mind that "retrieval" need not literally be a matter of retrieving content - this does not by itself imply that retrieved episodic memories are themselves contentless, since the states produced by retrieval might be imbued with content through the involvement of some other factor.

If this is right, then there need be no tension between radical enactivism about memory and an understanding of memory as a form of imagination, even if we grant that imagination produces contentful states. Consider the view defended recently by Sant'Anna and Michaelian (201x), who, building on antirepresentationalist views of perception (e.g., Travis 2004; Genone 2014), argue that various forms of episodic thought, including episodic memory and episodic imagination, are "silent" in the sense that they do not by themselves establish accuracy conditions and thus do not have content. On the view in question, content is acquired only through the "habits of action" that accompany the production of episodic thoughts, which include, in particular, "habits of judging" certain things to be the case about the episodes that are their objects. Moreover, because they argue that "genuinely remembering an event does not require the preservation of a trace connecting the actual memory to a previous perceptual state" (Sant'Anna and Michaelian 201x, 23), their view suggests that, even if remembering does not involve the retrieval of stored content, it is possible for retrieved memories to be imbued with content by other means.

Hutto and Peeters seem to hint at a similar view when they say that episodic memory judgements can have content, even if they deny that retrieved episodic memories themselves have content. While the details of their view are not entirely clear, the basic idea is that contentful judgements about an episode arise only with the mastery of narrative practices. Appealing to recent developments in developmental psychology, Hutto (2017; see also Hutto and Myin 2017, ch. 9) argues that the capacity to take a given episodic thought of an event to stand for - that is, to contentfully represent - a particular past event is a capacity that starts to develop only after the age of three, when children begin to engage in narrative constructions regarding past and future events with their parents. Studies suggest that it is only when these abilities are quite well-developed that we learn to associate episodic thoughts with our personal pasts (Nelson and Fivush 2004; Fivush and Nelson 2004; Hoerl 2007; Fivush 2011). Thus it seems that, on the radical enactivist view as well as Sant'Anna and Michaelian's view, episodic retrieval can be seen as involving a transition from a contentless to a contentful state, even if the production of the contentful state is an additional step. In short, contrary to the suggestion made by Hutto and Peeters, it does not seem to be the case that "[i]f all imagining is necessarily representational 
then defeat for radically enactive accounts of imagination and memory follows briskly" (2018, 110-111); the important question is, rather, whether remembering involves retrieval of stored content.

We will thus continue to take it that the core claim of radical enactivism with respect to episodic memory is NC-episodic, interpreted as we have been interpreting it so far: (episodic) memory traces are not contentful. Now, we have seen that Hutto and Peeters have two basic motivations for adopting a contentless conception of traces. First, they follow De Brigard (2014) in taking it to be required by considerations of empirical adequacy. Second, of course, such a conception fits nicely with the general antirepresentationalist thrust of radical enactivist theory. Recent causal and postcausal theorists have focused heavily on the philosophical implications of empirical research on reconstructive, simulative, or imaginative remembering and will be sympathetic to the former motivation. They will not necessarily be particularly sympathetic to the latterwhile there have been occasional displays of hostility to accounts of memory as a representational capacity, these have for the most part been motivated by fairly traditional arguments for direct realism or relationalism, rather than by anything like enactivist antirepresentationalism (Debus, 2008). Nevertheless, philosophers of memory have increasingly arrived at the same conclusion as Hutto and Peeters, albeit by a different route, adopting a contentless conception of traces not because they wish to dispense with content as such but rather because the widely-adopted distributed conception of traces has come to seem, when properly understood, to lead inexorably to the contentless conception. In the following section, we retrace the route they have taken, demonstrating, in the process, that an important subset of recent theories are compatible with the core features of radical enactivism about memory.

\section{Contentless memory traces and (post)causal theories of memory}

There are a few contemporary defenders of epistemic (James, 2017) and even empiricist (Byrne, 2010) theories of memory, but the overwhelming majority of philosophers of memory today endorse theories that recognizably descend from the causal theory of memory as formulated by Martin and Deutscher (Debus, 2017). It is on a subset of these that we focus here.

\subsection{The (neo)classical causal theory}

Perhaps the chief task of a philosophical theory of memory is to provide a general account of the features that distinguish remembering from other memory-like processes, including imagining. According to Martin and Deutscher's (1966) version of the causal theory - what Michaelian and Robins (2018) refer to as the "classical" causal theory-remembering is distinguished from mere imagining by the presence, in the case of remembering, and the absence, in the case of imagining, of an appropriate causal connection between a retrieved putative memory of an event and the remembering subject's earlier experience of that event. A causal connection, in turn, is taken to be "appropriate" just in case it is sustained by a memory trace originating in the subject's experience of the 
event. And a memory trace is assumed to be a contentful entity the content of which derives from the relevant experience.

The details of Martin and Deutscher's own account of the nature of the content of memory traces - their account, which compares memory traces to the grooves of a record, is emphatically local, in the sense introduced in section 3have not had much of an impact (see Robins 2017). Their general assumption that traces have content - of some sort - was, however, until recently taken for granted by most philosophers of memory. Bernecker, for example, in developing his "neoclassical" (Michaelian and Robins, 2018) version of the causal theory, follows Sutton in rejecting Martin and Deutscher's local account and adopting a connectionist-inspired distributed view of traces. Like Sutton, he argues that remembering does not involve the retrieval of discrete traces stored in discrete locations: "information is stored in the strengths of connections between neurons. ... [T]races are distributed rather than local: what can be distinctly remembered need not be held distinctly or independently, since each memory item is spread or 'superposed' across many elements in a neural network" (2010, 137). He continues, however, to take for granted that traces are contentful, asking: "[w] hat are the mechanisms by which traces store content?" (2010, 133).

Martin and Deutscher's influence continues to be felt here, not only in the explicit assumption that traces are contentful but also, arguably, in an implicit assumption that, while storage is distributed, content is in some sense local. ("Memory items" are distributed across multiple elements in a network, but they remain items.) This combination of views - storage format as distributed and stored content as local - is not unique to Bernecker, and we will return to it below. For now, we note that the (neo)classical causal theory is highly intuitive, encapsulating a natural picture of remembering on which it amounts to the transmission of content from experience to retrieved memory via the intermediary of a contentful memory trace. It is also, whatever its other merits, straightforwardly incompatible with $\mathrm{NC}$-episodic and thus with radical enactivism about memory. We will therefore set it aside in the remainder of this paper. $^{11}$

\subsection{The simulation theory}

Michaelian (2016b), in developing the simulation theory, positions himself in opposition to the causal theory, denying that appropriate causation is necessary for the occurrence of genuine remembering; the simulation theory can thus be seen as a postcausal theory (Michaelian and Robins, 2018). ${ }^{12}$ The positive argument for the simulation theory - negative arguments are discussed below-builds on research on memory as a form of mental time travel analogous to episodic future thought ${ }^{13}$ research that suggests that the process of remembering the past is executed by the same system as the process of imagining the future and, indeed, that carrying out the latter process may be the primary function of the system.

\footnotetext{
${ }^{11}$ Just as we do not aim to assess the viability of radical enactivism, we do not aim to assess that of the causal theory or the other theories of memory discussed below.

${ }^{12}$ Shanton and Goldman (2010) and De Brigard (2014) defend simulationist accounts of remembering but, unlike Michaelian, do not explicitly deal with the question of the necessity of appropriate causation. In addition to the simulation theory, the category of postcausal theories includes Fernández' (2018) functionalist theory and, arguably, James' (2017) version of the epistemic theory.

${ }^{13}$ See Perrin and Michaelian 2017 and Sant'Anna 2018 for reviews.
} 
Since imagining a future event trivially does not presuppose the existence of a causal connection between the subject's thought of the event and his experience of the event, this, in turn, suggests that remembering a past event likewise should not be taken to presuppose the existence of a causal connection between the subject's thought of the event and his experience of the event: the episodic memory system (which is perhaps in fact an "episodic construction system"; Michaelian 2016b) appears simply not to be designed in such a way that the presence of such a connection can be taken for granted in every case of genuine remembering. The simulation theory thus maintains that previous efforts to identify the features that distinguish remembering from mere imagining were misguided: according to the simulation theory, to remember the past just is to imagine it. ${ }^{14}$

Like radical enactivism, the simulation theory is motivated by an appreciation of the reconstructive character of remembering and denies that remembering necessarily involves the transmission of content from an experience of an event to a retrieved memory of the event. One might therefore expect it, unlike the (neo)classical causal theory, to be compatible with NC-episodic. Unlike radical enactivism, however, the simulation theory is not motivated by hostility to the idea of stored content. In fact, while the simulation theory denies that any content needs to be transmitted from an experience of an event to a later apparent memory of the event in order for the apparent memory to qualify as a genuine memory, it does not deny that retrieval draws on contentful memory traces. On the contrary, the simulation theorist argues that, just as imagining a future event is a constructive process that draws on stored content originating in the subject's experiences of various events, remembering a past event is a reconstructive process that draws on stored content originating in the subject's experiences of various events. A retrieved memory of a given event will oftenindeed usually - include retrieved content originating in the subject's experience of the remembered event but will normally also include content originating in the subject's experiences of other events, and, in some cases, a retrieved memory of a given event will include only content originating in the subject's experiences of other events, i.e., it will include no content originating in the subject's experience of the remembered event. In both kinds of case, the simulation theory asserts that episodic remembering involves the retrieval of stored content.

Thus, while the simulation theory is compatible with the second key feature of radical enactivism about memory (its emphasis on the reconstructive character of remembering), it is incompatible with its third key feature (NC), and Hutto and Peeters therefore take the simulation theory to be, like the (neo)classical causal theory, incompatible with their account, singling out the following passage as illustrative of the simulation theorist's commitment to the contrary of NC-episodic.

Declarative memories are available to consciousness and thus can affect the activities of other cognitive systems. I remember that it has been sunny every day for the past week; I infer that it will be

\footnotetext{
${ }^{14}$ This argument takes the mental time travel framework for granted and, moreover, rests on a "continuist" interpretation of that framework according to which it implies that, aside from their different temporal orientations, there is no difference in kind between episodic memory and episodic future thought. For alternative "discontinuist" interpretations of the mental time travel framework, see Perrin 2016 and Dokic and Arcangeli 2018. For an argument against the framework itself, see Mahr and Csibra 2018.
} 
sunny today; I therefore leave home without bringing an umbrella. An appeal to stored information is essential to the explanation of my behaviour in this case, for the memory does not cause the behaviour directly, but only via an intermediate process of reasoning. Conscious access to stored contents allows them to enter into the causation of behaviour in various indirect ways, via reasoning, imagination, and other routes; if the subject were not to have such access, declarative memory would be unable to provide inputs to other processes. Thus, were we to suppose that declarative memory does not store information, we would be unable to explain certain modifications in the behaviour of the organism. (Michaelian 2016b, 27-28; Hutto and Peeters' emphases)

We will come back to this passage in section 5. But we first want to show, in the remainder of section 4 , that, appearances to the contrary notwithstanding, the simulation theory can easily be made compatible with $\mathrm{NC}$-episodic, in the sense that the simulation theorist can say everything that he needs to say without any reference to stored content. We will show, moreover, that there is pressure for the simulation theorist to do so from within the community of causal and postcausal theories, for reasons independent of the sort of general antirepresentationalist considerations emphasized by radical enactivists.

One might wonder whether our argument is undermined by a discrepancy between the notion of content with which radical enactivists work and the notion with which philosophers of memory work. Radical enactivists understand content in semantic terms, that is, as being that in virtue of which a memory would stand for or be about something in the world. Philosophers of memory, in contrast, arguably understand it in componential terms (Perrin 2018), that is, as pertaining to the elements or building blocks in virtue of which a memory is experienced in a certain way; the idea is that if, for example, one remembers one's birthday party last year, one's memory will include various bits of information, such as "cake", "people", and "drinks", which determine, at least in part, how one experiences the memory. It is not entirely clear whether the fact that memory has content in the componential sense entails that it has content in the semantic sense. If it does, this would simply reinforce the argument for the negative claim that the classical causal theory and the simulation theory are not compatible with radical enactivism about memory. If it does not, this would undermine at most that argument; it would not undermine the argument, developed in the remainder of this section, for the positive claim that the procedural causal theory and a "nontransmissionist" version of the simulation theory are compatible with radical enactivism about memory: if the view that memory involves the storage and retrieval of content, as that view figures in the classical causal theory and the simulation theory, refers to content in the componential sense, then, because the fact that a given process involves componential content does not entail that it involves semantic content, the classical causal theory and the simulation theory might turn out be compatible with radical enactivism after all, just as - we argue below — the procedural causal theory and the nontransmissionist simulation theory are compatible with radical enactivism. Because the truth of the claim that the classical causal theory and the simulation theory are compatible with radical enactivism turns on the precise relationship between componential content and semantic content, we will 
not attempt to defend it here. The claim that the procedural causal theory and the nontransmissionist simulation theory are compatible with radical enactivism about memory does not depend on this relationship, as both of the theories in question deny that remembering involves the storage and retrieval of either componential or semantic content; it is this claim that we defend in the remainder of the section.

\subsection{The procedural causal theory}

Martin and Deutscher, we noted above, worked with a conception of traces as local and contentful. Their version of the causal theory has been enormously influential, but Sutton's (1998 and subsequent) arguments for the distributed conception of traces have convinced most (post)causal theorists to abandon the local conception. These theorists have, however, been slow to recognize that the distributed conception of traces may be in tension with the contentful conception of traces. ${ }^{15}$

One illustration of the failure to recognize this tension is provided by Bernecker's neoclassical causal theory, which, we have seen, combines the claim that remembering involves the storage of content with the claim that the format in which that content is stored is distributed. Another illustration is provided by a version of the causal theory previously defended by Michaelian. ${ }^{16}$ In order to account for the reconstructive character of remembering, including the fact that retrieved representations routinely include content not included in the content of the corresponding earlier experiences, Michaelian (2011) combines a causal theory similar to Martin and Deutscher's with Sutton's distributed conception of traces. The resulting "constructive causal theory" continues to take the contentful conception of traces for granted, treating storage as distributed but content as effectively local. The constructive causal theory thus operates with a "componential" understanding of causation (Perrin, 2018), an understanding on which the causal link (allegedly) necessary for the occurrence of genuine remembering is to be analyzed in terms of the transmission of discrete "packets" of content from experience to retrieved representation. The packets of content left behind by experience can, in Michaelian's view, be shaped during encoding and consolidation, modified by ongoing experience during reconsolidation, and transformed and recombined in various ways during retrieval, thus accounting for a range of aspects of reconstruction. But experience is still seen as leaving behind discrete contents that can be transmitted, in one form or another, to retrieved memories. This goes as well for the simulation theory, which denies that remembering a given event presupposes the transmission of content from experience of the particular event remembered but continues to treat remembering as involving the transmission of content from experience in general.

That the package of views that Bernecker and Michaelian each offers uscombining the distributed conception of traces with the contentful conceptionmay be incoherent has recently started to become clear. Perrin (2018), in partic-

\footnotetext{
${ }^{15}$ This slowness may be due to a failure on the part of philosophers of memory to take older debates over the storage of content in connectionist networks - in which some (e.g., Ramsey et al. 1990) argued that the lack of storage of explicit content in such networks meant that they simply did not store content, while others (e.g., O'Brien 1991) argued that they might nevertheless store implicit content-into account.

${ }^{16}$ See Robins 2016a for a recent approach in the same general spirit.
} 
ular, has argued that, once we adopt the distributed conception, we are bound to abandon the componential understanding of causation in memory in favour of an alternative "procedural" understanding in line with De Brigard's characterization of traces as dispositions. In order to illustrate the nature of the causal link that may obtain between a (contentful) retrieved memory and a (contentful) earlier experience even in the absence of stored content, Perrin offers the following metaphor.

Let's imagine you have two copies of one and the same puzzle. The pieces are exactly the same in number, forms, and pictures in each box. Obviously, however alike the pieces are, as one makes one of the two puzzles one does not use the pieces of the other. In other terms, the two series of construction operations apply respectively to causally unrelated (though similar) bits of representation. But at the same time ...these construction operations themselves can be causally related. For instance, if you make the two puzzles one after the other, you will possibly perform the second time better than the first time. And should you repeat the operations further, the enhancement will probably get ever clearer. So arguably, an earlier series of construction operations can get a later series enhanced, while the manipulated sets of pieces are distinct and causally unrelated. $(2018,37)$

The basic idea is that, if (in line with views such as that of O'Regan and Noë 2001) we think of perceiving as a constructive process, and if (in line with research on mental time travel) we think of remembering as a reconstructive process, then we can acknowledge that the latter process is (when genuine remembering occurs) causally dependent on the former process - for example, in that it manifests greater fluency - despite the fact that no component of the representation constructed during perception is transmitted to the representation reconstructed during retrieval. ${ }^{17}$

Perrin claims not just that we can account for causation in the absence of the transmission of content but also that, given the distributed conception of traces, we must do so. We noted in section 3 that the relationship between Sutton's distributed conception of traces and the contentless conception that Hutto and Peeters find in De Brigard was not entirely clear. Perrin's procedural causal theory, which claims that appropriate causation is necessary for the occurrence of genuine remembering but understands causation in procedural rather than componential terms, helps to clarify the matter. What Perrin argues is that the distributed conception must be understood as a purely dispositional conception and hence as necessitating the contentless conception: if the laying down of a memory traces amounts simply to the modification of the strengths of connections among nodes in a network, then all there is, between encoding and retrieval, is a disposition to produce a representation with a certain content; and if all there is is a disposition to produce a representation with a certain content, then, strictly speaking, no content is stored.

\footnotetext{
${ }^{17}$ One might worry here that the puzzle analogy allows the procedural causal theorist to smuggle into his account the very (componential) content that he rejects (in the form of the puzzle pieces). The procedural causal theorist would presumably argue, in response, that the components of the content of the retrieved representation are generated at the time of retrieval by the reactivation of areas that were activated during experience.
} 
The procedural causal theorist adopts the distributed conception of traces and therefore adopts the contentless conception. The neoclassical and constructive variants of the causal theory discussed above, as well as the simulation theory, attempt to have things both ways by combining the distributed conception with the contentful conception. If Perrin is right, this cannot be done. Though Sutton himself has sometimes referred to distributed traces as having "implicit" content, this is misleading at best. Distributed content is no content at all; the contentful conception in effect entails the local conception. The neoclassical causal theory, the constructive causal theory, and the simulation theory are, as far as the nature of memory traces is concerned, incoherent.

\subsection{A nontransmissionist simulation theory}

Assuming that Perrin is right, we might wonder why it took so long for this incoherence to become apparent. ${ }^{18}$ The explanation appears to be, at least in part, simply that the attention of (post)causal theorists has been directed elsewhere. They have, in particular, been preoccupied with a debate between views known as "preservationism" and "generationism", a debate that has been conducted in terms that have made it natural for defenders of both positions to take a contentful conception of traces for granted. ${ }^{19}$ According to preservationism (endorsed, e.g., by Bernecker 2010), it is incompatible with the occurrence of genuine remembering that the content of the retrieved (apparent) memory exceeds the content of the corresponding earlier experience. According to generationism (endorsed by Michaelian 2011 and 2016b and Perrin 2018), this is not the case: memory can tell one more than one experienced, in the sense that the content of a retrieved genuine memory of an event may sometimes exceed the content of the subject's experience of the event.

Preservationism is naturally described as the view that memory can only "preserve" the content of experience. Consequently, it has been natural for generationists to argue for their view by adducing cases (e.g., boundary extension; Intraub et al. 1992) in which memory appears not only to preserve the content of experience but also to generate new content. The result is that both preservationists and generationists have ended up taking "transmissionism" - the claim that content is transmitted from experience to retrieval via memory traces (Michaelian and Robins, 2018) — for granted. The preservationism/generationism and transmissionism/nontransmissionism distinctions are, however, orthogonal to one another: the former pair of views pertains to the relationship between the contents of the representations that exist at the beginning- and end-points of the process of remembering, while the latter pertains to whether there is content in play between these points (during storage). Thus one might coherently be

\footnotetext{
${ }^{18}$ In addition to Perrin (2018) and De Brigard (2014), Robins (2016b) and Cheng and Werning (2016) have hinted at the necessity for a contentless conception of traces. Going further back, there are few clear discussions of the issue, though Vosgerau (2010) does presage recent developments in some respects.

${ }^{19}$ The preservationism/generationism debate is multifaceted, and "preservationism" and "generationism" have been used to refer to a variety of pairs of views related to but distinct from those discussed here. See, e.g., Fernández (2016) on whether episodic memory generates (second-order) knowledge of the source of one's (first-order) knowledge in past experience (cf. Dokic 2014) and Lackey (2005) and Senor (2007) on whether memory generates knowledge via the coming and going of defeaters. Frise (2017) and Bernecker and Grundmann (201x) discuss additional varieties of preservationism/generationism.
} 
either a preservationist or a generationist while being either a transmissionist or a nontransmissionist. Bernecker (2010), for example, combines transmissionism with preservationism, while Michaelian (2011; 2016b) combines transmissionism with generationism. Perrin (2018) endorses generationism but rejects transmissionism in favour of nontransmissionism. Similarly, though it is not obvious how this combination of views might be motivated, one might in principle combine preservationism and nontransmissionism, maintaining that there is no content in play during storage but that the occurrence of genuine remembering nevertheless requires that the content recreated at the time of retrieval not exceed the content created at the time of perception.

Although Michaelian endorses transmissionism, the simulation theorist can in fact follow the procedural causal theorist in rejecting transmissionism and thereby endorsing the contentless conception of traces and NC-episodic. There are two basic arguments for the simulation theory. The first is the positive argument reviewed above. This argument appeals to the relationship between remembering the past and imagining the future and makes no essential reference to the transmission of content. A nontransmissionist simulation theorist would thus be able to run the argument without modification. The second, negative argument - really a pair of arguments - is designed to show that, contra (neo)classical, constructive, and procedural causal theories, appropriate causation is neither necessary nor sufficient for the occurrence of genuine remembering. These "anti-necessity" and "anti-sufficiency" arguments are conveniently formulated in terms of the transmission of content, but they can be modified so as to be made consistent with a nontransmissionist simulation theory.

Consider, first, the anti-sufficiency argument. Causal theorists of all stripes maintain that, assuming that the earlier experience and the retrieved representation are both accurate with respect to the event, satisfaction of a condition requiring the presence of an appropriate causal connection between a subject's retrieved representation and his earlier experience of an event is sufficient for the retrieved representation to qualify as a memory of the event. A causal connection, again, is taken to be appropriate just in case it is sustained by a memory trace originating in the subject's experience of the event. Transmissionist causal theorists understand the role played by the trace as being the transmission of content from the earlier experience to the retrieved representation. The basic idea of the anti-sufficiency argument, as formulated by the transmissionist simulation theorist, is that (in line with research on the reconstructive character of remembering of the sort reviewed by Michaelian 2016b) contents originating in experiences of multiple earlier events may contribute to the content of a single retrieved representation, whether because they make their way into the trace resulting from the experience of the remembered event (during encoding, consolidation, or reconsolidation) or because they result in distinct traces that themselves, along with a trace originating in experience of the remembered event, directly contribute content to the retrieved representation (during retrieval). When this occurs, a single present representation is "appropriately" causally connected to multiple earlier experiences. Together with the fact that the retrieved representation might be accurate with respect to more than one of the relevant events - consider, for example, a memory of an event of a type that the subject has experienced many times - this implies that a single retrieved representation might count as a memory of multiple earlier events. There may, of course, be memories that refer to multiple events, but episodic memories, in 
particular, have a singular character, so we may bracket these here. The argument concludes that satisfaction of the appropriate causation condition is not sufficient for the occurrence of genuine memory.

This argument can be reformulated in nontransmissionist terms. Nontransmissionist causal theorists understand the role played by the trace as being a matter of sustaining a disposition to produce a content similar to that of the experience of the remembered event when given a suitable cue. The basic idea of the anti-sufficiency argument, as formulated by the nontransmissionist simulation theorist, is, again, that a single retrieved representation might be "appropriately" causally connected to multiple earlier experiences. Given that a trace is not an entity but rather a disposition underwritten by connection strengths among nodes in a network, there will not normally be a one-to-one correspondence between experiences and traces. Instead, multiple experiences are normally responsible for the strength and continuing existence of a given disposition, as connection strengths are reinforced and weakened during encoding, consolidation, and reconsolidation. Consider, again, the workings of connectionist networks: in virtue of the connection strengths among its nodes, a network can be said to have a disposition to produce a certain output (corresponding to retrieval) when given a certain input (corresponding to the retrieval cue), but these connection strengths are the product of multiple training sessions (corresponding to multiple experiences). If there is not a one-to-one correspondence between experiences and traces, there cannot be a one-to-one correspondence between retrieved representations and experiences, implying that a single retrieved representation will sometimes be "appropriately" causally connected to multiple earlier experiences. As before, this leads quickly to the conclusion that satisfaction of the appropriate causation condition is not sufficient for the occurrence of genuine memory. ${ }^{20}$

Turning to the anti-necessity argument, causal theorists maintain that satisfaction of the appropriate causation condition is necessary for a retrieved representation to qualify as a genuine memory of the apparently remembered event. The basic idea of the anti-necessity argument, as formulated by the transmissionist simulation theorist, is that the reconstructive character of remembering suggests that, even when the memory (or episodic construction) system functions exactly as it should, the content of a retrieved representation need not be

\footnotetext{
${ }^{20} \mathrm{~A}$ comparison of the transmissionist and nontransmissionist versions of the anti-sufficiency argument suggests two observations. The first is that distributed and procedural causal theorists have yet to really offer an account of appropriate causation. Given the local conception of traces, there might in principle be a one-to-one correspondence between traces and experiences, even if, in practice, there is always always a one-to-many correspondence. Given the distributed conception, in contrast, there is a one-to-many correspondence virtually by definition. The second is that, while the one-to-many correspondence between traces and experiences ensures that the the nontransmissionist simulationist can come to the same conclusion as the transmissionist simulationist regarding the insufficiency of appropriate causation, the structure of the nontransmissionist anti-sufficiency argument does not precisely mirror that of the transmissionist anti-sufficiency argument. The latter turns on the claim that distinct contents originating in experiences of distinct events can make their way into a single retrieved memory. The former replaces this claim with the claim that retrieval of a single memory can be treated as the activation of a disposition originating in experiences of distinct events. In order for the nontransmissionist argument to mirror the transmissionist argument, it would need, instead, to appeal to the claim that retrieval of a single memory can be treated as the activation of distinct dispositions originating in experiences of distinct events. Whether this claim is coherent cannot be determined without a more detailed discussion of the nature of the relevant dispositions than we can feasibly undertake here.
} 
transmitted, even in part, from the content of the relevant earlier experience. Reconstruction during encoding, consolidation, reconsolidation, and retrieval implies that the content of a retrieved representation will never or essentially never be transmitted exclusively from the content of a single experience. In some cases, a majority of the content may be so transmitted, but, in some cases, only a minority may be so transmitted. Given that the same reconstructive process is at work both when a majority of the content of the retrieved representation is transmitted from the content of the experience of the apparently remembered event and when a minority of the content of the retrieved representation is transmitted from the content of the apparently remembered event, the simulation theorist invites us to conclude that the subject is genuinely remembering in both cases. He then invites us to go one step further: given what we know about how the memory (or episodic construction) system works, we have to assume that the same reconstructive process may be at work even when none of the content of the retrieved representation is transmitted from the content of the experience of the apparently remembered event. And this suggests that a retrieved representation may qualify as a genuine memory of the apparently remembered event even when no content at all is transmitted from experience to retrieval. If this is right, then satisfaction of the appropriate causation condition (understood in transmissionist terms) is not necessary for memory.

This argument, too, can be reformulated in nontransmissionist terms. We have already seen that, given nontransmissionism, there cannot be a one-to-one correspondence between experiences and traces, simply because the dispositions that are traces are not in general the products of single experiences. This means that there will normally be a causal connection of the relevant sort not just between a given retrieved representation and a single earlier experience but also between that representation and other experiences. In some cases, few other experiences may contribute to shaping the disposition, but, in other cases, the contribution of the experience of the relevant event may be dwarfed by the contributions of other experiences - again, consider a memory for an event of a type that the subject has experienced many times. Given that the same process is at work in cases of both sorts, we should say that genuine memory occurs in cases of both sorts. Now, it may be that, given the distributed conception of traces, there is almost always a causal connection of the relevant sort between any given retrieved representation and any given earlier experience. It is to be expected, however, that there are cases in which there is no causal connection between a given representation and a given earlier experience - this may, for example, occur when the encoding is interrupted for one reason or another. ${ }^{21}$ And this means that it is to be expected that there are cases in which the experience of the relevant event makes no contribution at all to the shaping of the disposition responsible for the production of a retrieved representation. Given what we know about the reconstructive workings of the memory system, we have to assume that the same process may be at work even in cases of this sort. As before, this brings us to the conclusion that satisfaction of the appropriate causation condition (now understood in nontransmissionist terms) is not necessary for memory.

\footnotetext{
${ }^{21}$ If we deny the possibility of such cases, insisting that there is always a causal connection, then the necessity condition collapses into triviality.
} 
If he is to avoid the implication that any imagining of an event whatsoever qualifies as a memory of the event, the simulation theorist will need to provide a substitute of some sort for the appropriate causation condition. Michaelian (2016b) has argued that a reliability condition - which might be satisfied even when the causal condition is not - enables us to distinguish between genuine memory and instances of merely apparent memory such as misremembering and confabulation. The suitability of a reliability condition has, unsurprisingly, been challenged by defenders of the causal theory such as Robins (201xa; 201xb) and Bernecker (2017), ${ }^{22}$ but this debate need not concern us here, as our aim is not to assess the viability of the simulation theory but rather to determine whether the simulation theorist's arguments are compatible with nontransmissionism. And we have seen that, while the anti-necessity and anti-sufficiency arguments are conveniently formulated in terms of the transmission of content, they can be modified so as to be made consistent with nontransmissionism. We thus conclude that, while the transmissionist simulation theory formulated by Michaelian is incompatible with NC-episodic, transmissionism is not essential to the simulation theory.

\section{After transmissionism: Kinds of memory (er- ror)}

A nontransmissionist simulation theory will be compatible with NC-episodic. We have thus seen that, while the (neo)classical and constructive causal theories are straightforwardly incompatible with radical enactivism about memory, the simulation theory can be made compatible with radical enactivism, and the procedural causal theory is compatible with radical enactivism as it stands. ${ }^{23}$ This brings us to our conclusion: while other mainstream theories would need to be assessed separately, certain causal theories (namely, the procedural causal theory) and postcausal theories (namely, a nontransmissionist version of the simulation theory) can take NC-episodic on board, despite the fact that they are not motivated by the sort of general hostility to mental content that animates radical enactivism. These theories are thus compatible with all three key features of radical enactivism about memory.

We have left one thread hanging: the status of NC-semantic. The theories on which we are focusing themselves focus on episodic memory. For the reasons given above, if they adopt NC-episodic, procedural causal and simulation theorists will presumably also adopt NC-semantic. But, in conjunction with another thread that we have left hanging, namely, the status of Michaelian's argument in the passage quoted on page 15 , this raises a question about the implications of NC for the question of the boundaries of memory. Hutto and Peeters (2018, 113) take the passage in question to signal the simulation theorist's commitment to the contrary of NC. Taken in context, however, the point of the passage is not to argue against $\mathrm{NC}$, the contentless conception of traces, or nontransmission-

\footnotetext{
${ }^{22}$ See Michaelian 2016a, 201x for a response.

${ }^{23}$ That the procedural causal theory and the simulation theory are both compatible with radical enactivism about memory does not imply that there is no significant disagreement between them: the procedural causal theory remains a causal theory, and the procedural causal theorist will need to find a way of resisting (the nontransmissionist versions of) the simulation theorist's anti-necessity and anti-sufficiency arguments.
} 
ism. The point is, rather, to argue for a difference in kind between declarative memory and procedural memory; and a parallel argument can be run even if we adopt $\mathrm{NC}$, the contentless conception, and nontransmissionism.

As Tulving (2007) has pointed out, the term "memory" has tended to be used somewhat indiscriminately. Klein (2015), in fact, has argued that the term has been applied so widely that it has effectively been emptied of meaning. Appealing to a variety of empirical and theoretical considerations, he suggests that it ought to be applied much more narrowly: since episodic memory alone involves autonoetic consciousness - a phenomenal sense of the self in subjective timeonly episodic memory merits the name "memory"; the presence of autonoesis marks a fundamental distinction between episodic memory and everything else that has been called "memory", including semantic memory and procedural memory. In reply, Michaelian (2015) has argued that, if we want to carve memory at its joints, the distinctive feature of memory proper should be taken to be not the involvement of autonoesis but rather the involvement of encoding, storage, and retrieval of content. By this criterion, the fundamental distinction is - assuming transmissionism - not between episodic memory, on the one hand, and semantic and procedural memory, on the other hand, but rather between declarative memory, including both episodic and semantic memory, on the one hand, and procedural memory, on the other hand. ${ }^{24}$

This approach is, as noted in section 3, incompatible with NC, which says that neither declarative nor procedural memory involves the encoding, storage, and retrieval of content. But a parallel argument can be run even if we adopt $\mathrm{NC}$ /nontransmissionism. As we have emphasized, nontransmissionists can deny that encoding results in a contentful entity that is then stored and retrieved while accepting that "retrieval" nevertheless itself results in a contentful state. A nontransmissionist is thus free to say that the distinctive feature of memory is not the encoding, storage, and retrieval of content but rather the "retrieval" of content, where retrieval refers to the construction of a contentful representation that corresponds to a past experience and even (perhaps) causally depends on it via a dispositional trace it leaves behind but that does not include any content transmitted (via a contentful trace) from the experience. Since only declarative memory involves the construction of contentful representations, we can still conclude that only declarative memory is really memory, i.e., that the fundamental distinction is between declarative memory, on the one hand, and procedural memory, on the other hand. In short, even if we acknowledge that declarative remembering has (as Perrin would put it) a procedural character, we can still acknowledge a difference in kind between declarative memory and procedural memory. This is in accord with the claim, defended in section 3, that radical enactivism is not necessarily at odds with the view that retrieved semantic and episodic memories are contentful. As long as the acquisition of content by declarative memories amounts to an additional step-perhaps due to the construction of narratives, as suggested by Hutto (2017), or perhaps due to accompanying habits to produce judgements about episodes, as suggested by Sant'Anna and Michaelian (201x) - a nontransmissionist can hold on to the idea that declarative and procedural memory differ in kind without appealing to a

\footnotetext{
${ }^{24}$ Strictly speaking, Michaelian's view is that the fundamental distinction is between "cognitive" and "noncognitive" memory, terms that do not map precisely on to "declarative" and "nondeclarative". This subtlety does not affect our argument.
} 
difference between them with respect to retrieval of stored content. ${ }^{25}$

Before concluding, we respond to a general worry about the contentless conception of traces. One might be concerned that, if, as the contentless conception implies, "retrieval" is not literally retrieval, not only in the sense that it is a reconstructive process but also in the sense that there is no stored content out of which a representation might be reconstructed, memory traces no longer play any clear role in explaining the correspondence between the retrieved representation and the original experience. In response to this worry, we observe that the explanatory role assigned to traces by the procedural causal theory is reasonably clear: the original experience of the remembered event results in a disposition - the contentless trace - the activation of which is responsible for the production of the (contentful) representation of the event at retrieval; the trace is thus the primary ingredient of the explanation of the subject's present ability to accurately represent a past event. The explanatory role assigned to traces by the nontransmissionist simulation theory is equally clear, though slightly less straightforward: multiple experiences - potentially but not necessarily including experience of the remembered event-result in a disposition - a contentless trace - the activation of which is responsible for the production of a (contentful) representation of an event at retrieval; the trace is thus one ingredient of the explanation of the subject's present ability to represent a past event, though it does not by itself explain the accuracy of the representation, since the simulationist must, given that the trace may not originate even in part in experience of the relevant event and will in general, even when it does originate in part in experience of the relevant event, originate also in experience of other events, additionally invoke the reliability of the principles governing the retrieval process. More work would, of course, need to be done to establish that either the procedural causal theory or the nontransmissionist simulation theory is correct, but this response should suffice to address the worry that, if one or the other of these theories is correct, traces no longer play a clear explanatory role.

By way of conclusion, we note that radical enactivism may have implications not only for the question of how to distinguish among kinds of memory but also for the question of how to distinguish among kinds of memory error. Appealing to a constructive causal theory of memory, Robins, for example, argues that the distinction between confabulation (which occurs, very roughly, when a subject who is unable to remember a past event instead "makes up" a more or less plausible event) and misremembering (which occurs, for example, when a subject who has studied a list of thematically-related words mistakenly remembers a non-studied but thematically-consistent word as having appeared on the list) is a matter of the retention of information: in confabulation, no information is retained from the apparently remembered event; in misremembering, in contrast, "[the subject's] report is inaccurate, yet this inaccuracy is explicable only on the assumption that she has retained information from the event her representation

\footnotetext{
${ }^{25}$ One might wonder whether a nontransmissionist simulation theorist can really say everything that Michaelian says in the passage quoted on page 15. The same thing goes for nontransmissionist theorists in general: it is unclear how a procedural causal theorist, for example, can say everything that we need to say about the ways in which memory feeds into other cognitive processes and leads to behaviour without making any reference at all to stored content. We grant that this is unclear and indeed take it to be a major challenge for nontransmissionism. Our goal in this section, however, has not been to show how nontransmissionists might meet that challenge but only (more modestly) to show how they might coherently adopt something like Michaelian's approach to the taxonomy of memory.
} 
mischaracterizes" (2016a, 434; cf. 201xa; 201xb). Appealing to the simulation theory of memory, Michaelian (2016a; 201x) has argued, in opposition to Robins, that the distinction is, rather, a matter of reliability: in confabulation, the subject remembers unreliably; in misremembering, the subject remembers reliably but nevertheless ends up with an inaccurate memory. Since it denies - in opposition to the constructive causal theory - that remembering involves the retention of information, radical enactivism is incompatible with Robins' approach, but it is - assuming a nontransmissionist version of the simulation theory of the sort sketched in section 4-perfectly compatible with Michaelian's approach. Radical enactivism may thus provide additional ammunition for simulation theorists in their ongoing debate with causal theorists.

\section{References}

Adams, F. and Aizawa, K. (2008). The Bounds of Cognition. Wiley.

Arcangeli, M. (201x). Supposition and the Imaginative Realm: A Philosophical Inquiry. Routledge. Forthcoming.

Bernecker, S. (2010). Memory: A Philosophical Study. Oxford University Press.

Bernecker, S. (2017). A causal theory of mnemonic confabulation. Frontiers in psychology, 8:1207.

Bernecker, S. and Grundmann, T. (201x). Knowledge from forgetting. Philosophy and Phenomenological Research. Forthcoming.

Byrne, A. (2010). Recollection, perception, imagination. Philosophical Studies, 148(1):15-26.

Campbell, D. (2014). Review of the book Radicalizing Enactivism by D. D. Hutto and E. Myin. Analysis, 74:174-176.

Cheng, S. and Werning, M. (2016). What is episodic memory if it is a natural kind? Synthese, 193(5):1345-1385.

Clark, A. and Chalmers, D. (1998). The extended mind. Analysis, 58(1):7-19.

Colombo, M. (2014). Explaining social norm compliance. A plea for neural representations. Phenomenology and the Cognitive Sciences, 13(2):217-238.

De Brigard, F. (2014). Is memory for remembering? Recollection as a form of episodic hypothetical thinking. Synthese, 191(2):155-185.

Debus, D. (2008). Experiencing the past: A relational account of recollective memory. Dialectica, 62(4):405-432.

Debus, D. (2017). Memory causation. In Bernecker, S. and Michaelian, K., editors, The Routledge Handbook of Philosophy of Memory, pages 63-75. Routledge.

Dokic, J. (2014). Feeling the past: A two-tiered account of episodic memory. Review of Philosophy and Psychology, 5(3):413-426. 
Dokic, J. and Arcangeli, M. (2018). Affective memory: A little help from our imagination. In Michaelian, K., Debus, D., and Perrin, D., editors, New Directions in the Philosophy of Memory, pages 139-157. Routledge.

Fernández, J. (2016). Epistemic generation in memory. Philosophy and Phenomenological Research, 92(3):620-644.

Fernández, J. (2018). The functional character of memory. In Michaelian, K., Debus, D., and Perrin, D., editors, New Directions in the Philosophy of Memory, pages 52-72. Routledge.

Fivush, R. (2011). The development of autobiographical memory. Annual Review of Psychology, 62:559-582.

Fivush, R. and Nelson, K. (2004). Culture and language in the emergence of autobiographical memory. Psychological Science, 15(9):573-577.

Frise, M. (2017). Preservationism in the epistemology of memory. The Philosophical Quarterly, 67(268):486-507.

Genone, J. (2014). Appearance and illusion. Mind, 123(490):339-376.

Gładziejewski, P. (2015). Explaining cognitive phenomena with internal representations: A mechanistic perspective. Studies in Logic, Grammar and Rhetoric, 40(1):63-90.

Goldman, A. (2006). Simulating Minds: The Philosophy, Psychology, and Neuroscience of Mindreading. Oxford University Press.

Hoerl, C. (2007). Episodic memory, autobiographical memory, narrative: On three key notions in current approaches to memory development. Philosophical Psychology, 20(5):621-640.

Hurley, S. (1998). Consciousness in Action. Harvard University Press.

Hutto, D. D. (2017). Memory and narrativity. In Bernecker, S. and Michaelian, K., editors, The Routledge Handbook of Philosophy of Memory, pages 192-204. Routledge.

Hutto, D. D. and Myin, E. (2013). Radicalizing Enactivism: Basic Minds Without Content. MIT Press.

Hutto, D. D. and Myin, E. (2017). Evolving Enactivism: Basic Minds Meet Content. MIT Press.

Hutto, D. D. and Peeters, A. (2018). The roots of remembering: Radically enactive recollecting. In Michaelian, K., Debus, D., and Perrin, D., editors, New Directions in the Philosophy of Memory, pages 97-118. Routledge.

Intraub, H., Bender, R. S., and Mangels, J. A. (1992). Looking at pictures but remembering scenes. Journal of Experimental Psychology: Learning, Memory, and Cognition, 18(1):180-191. 
Irish, M. (2016). Semantic memory as the essential scaffold for future-oriented mental time travel. In Michaelian, K., Klein, S. B., and Szpunar, K. K., editors, Seeing the Future: Theoretical Perspectives on Future-Oriented Mental Time Travel, pages 389-408. Oxford University Press.

James, S. (2017). Epistemic and non-epistemic theories of remembering. Pacific Philosophical Quarterly, 98:109-127.

Klein, S. B. (2015). What memory is. Wiley Interdisciplinary Reviews: Cognitive Science, 6(1):1-38.

Klein, S. B., Loftus, J., and Kihlstrom, J. F. (2002). Memory and temporal experience: The effects of episodic memory loss on an amnesic patient's ability to remember the past and imagine the future. Social Cognition, 20(5):353379 .

Lackey, J. (2005). Memory as a generative epistemic source. Philosophy and Phenomenological Research, 70(3):636-658.

Loader, P. (2013). Is my memory an extended notebook? Review of Philosophy and Psychology, 4(1):167-184.

Mahr, J. B. and Csibra, G. (2018). Why do we remember? The communicative function of episodic memory. Behavioral and Brain Sciences, 41:e1.

Martin, C. B. and Deutscher, M. (1966). Remembering. The Philosophical Review, 75(2):161-196.

Michaelian, K. (2011). Generative memory. Philosophical Psychology, $24(3): 323-342$.

Michaelian, K. (2012). Is external memory memory? Biological memory and extended mind. Consciousness and Cognition, 21(3):1154-1165.

Michaelian, K. (2015). Opening the doors of memory: Is declarative memory a natural kind? Wiley Interdisciplinary Reviews: Cognitive Science, 6(6):475482 .

Michaelian, K. (2016a). Confabulating, misremembering, relearning: The simulation theory of memory and unsuccessful remembering. Frontiers in Psychology, $7: 1857$.

Michaelian, K. (2016b). Mental Time Travel: Episodic Memory and Our Knowledge of the Personal Past. MIT Press.

Michaelian, K. (2018). Episodic and semantic memory and imagination: The need for definitions. American Journal of Psychology, 131(1):99-103. Review essay on M. S. Humphreys and K. A. Chalmers, Thinking about Human Memory.

Michaelian, K. (201x). Confabulating as unreliable imagining: In defence of the simulationist account of unsuccessful remembering. Topoi. Forthcoming. 
Michaelian, K., Perrin, D., and Sant'Anna, A. (201x). Continuities and discontinuities between imagination and memory: The view from philosophy. In Abraham, A., editor, The Cambridge Handbook of Imagination. Cambridge University Press. Forthcoming.

Michaelian, K. and Robins, S. K. (2018). Beyond the causal theory? Fifty years after Martin and Deutscher. In Michaelian, K., Debus, D., and Perrin, D., editors, New Directions in the Philosophy of Memory, pages 13-32. Routledge.

Myin, E. and Hutto, D. D. (2015). REC: Just radical enough. Studies in Logic, Grammar and Rhetoric, 41(1):61-71.

Nelson, K. and Fivush, R. (2004). The emergence of autobiographical memory: A social cultural developmental theory. Psychological Review, 111(2):486.

Noë, A. (2004). Action in Perception. MIT press.

O'Brien, G. J. (1991). Is connectionism commonsense? Philosophical Psychology, 4(2):165-178.

O'Regan, J. K. and Noë, A. (2001). A sensorimotor account of vision and visual consciousness. Behavioral and Brain Sciences, 24(5):939-973.

Perrin, D. (2016). Asymmetries in subjective time. In Michaelian, K., Klein, S. B., and Szpunar, K. K., editors, Seeing the Future: Theoretical Perspectives on Future-Oriented Mental Time Travel, pages 39-61. Oxford University Press.

Perrin, D. (2018). A case for procedural causality in episodic recollection. In Michaelian, K., Debus, D., and Perrin, D., editors, New Directions in the Philosophy of Memory, pages 33-51. Routledge.

Perrin, D. and Michaelian, K. (2017). Memory as mental time travel. In Bernecker, S. and Michaelian, K., editors, The Routledge Handbook of Philosophy of Memory, pages 228-239. Routledge.

Ramsey, W., Stich, S., and Garan, J. (1990). Connectionism, eliminativism, and the future of folk psychology. In Cole, D. J., Fetzer, J. H., and Rankin, T. L., editors, Philosophy, Mind, and Cognitive Inquiry: Resources for Understanding Mental Processes, pages 117-144. Springer.

Roberts, T. (2013). Review of Radicalizing Enactivism by D. D. Hutto and E. Myin. Notre Dame Philosophical Reviews. http://ndpr.nd.edu/news/40035radicalizing-enactivism-basicminds- without-content/. Accessed: 04/06/2018.

Robins, S. (2016a). Misremembering. Philosophical Psychology, 29(3):432-447.

Robins, S. (2016b). Representing the past: Memory traces and the causal theory of memory. Philosophical Studies, 173(11):2993-3013.

Robins, S. (2017). Memory traces. In Bernecker, S. and Michaelian, K., editors, The Routledge Handbook of Philosophy of Memory, pages 76-887. Routledge.

Robins, S. (201xa). Confabulation and constructive memory. Synthese. Forthcoming. 
Robins, S. K. (201xb). Mnemonic confabulation. Topoi. Forthcoming.

Sant'Anna, A. (2018). Mental time travel and the philosophy of memory. Unisinos Journal of Philosophy, 19(1):52-62.

Sant'Anna, A. and Michaelian, K. (201x). Thinking about events: A pragmatist account of the objects of episodic hypothetical thought. Review of Philosophy and Psychology. Forthcoming.

Senor, T. D. (2007). Preserving preservationism: A reply to Lackey. Philosophy and Phenomenological Research, 74(1):199-208.

Shanton, K. and Goldman, A. (2010). Simulation theory. Wiley Interdisciplinary Reviews: Cognitive Science, 1(4):527-538.

Shapiro, L. (2014). Review of Radicalizing Enactivism by D. D. Hutto and E. Myin. Mind, 123:213-220.

Sutton, J. (1998). Philosophy and Memory Traces: Descartes to Connectionism. Cambridge University Press.

Sutton, J. (2010). Exograms and interdisciplinarity: History, the extended mind, and the civilizing process. In Menary, R., editor, The Extended Mind, pages 189-225. MIT Press.

Sutton, J. and Williamson, K. (2014). Embodied remembering. In Shapiro, L., editor, The Routledge Handbook of Embodied Cognition, pages 315-325. Routledge.

Szpunar, K. K., Spreng, R. N., and Schacter, D. L. (2016). Toward a taxonomy of future thinking. In Michaelian, K., Klein, S. B., and Szpunar, K. K., editors, Seeing the Future: Theoretical Perspectives on Future-Oriented Mental Time Travel, pages 21-35. Oxford University Press.

Thompson, E. (2007). Mind in Life: Biology, Phenomenology, and the Sciences of Mind. Harvard University Press.

Travis, C. (2004). The silence of the senses. Mind, 113(449):57-94.

Tulving, E. (1972). Episodic and semantic memory. In Tulving, E. and Donaldson, W., editors, Organization of Memory, pages 381-402. Academic Press.

Tulving, E. (2005). Episodic memory and autonoesis: Uniquely human? In Terrace, H. S. and Metcalfe, J., editors, The Missing Link in Cognition: Origins of Self-Reflective Consciousness, pages 3-56. Oxford University Press.

Tulving, E. (2007). Are there 256 different kinds of memory? In Nairne, J. S., editor, The Foundations of Remembering: Essays in Honor of Henry L. Roediger III, pages 39-52. Psychology Press.

Varela, F., Thompson, E., and Rosch, E. (1991). The Embodied Mind: Cognitive Science and Human Experience. MIT press.

Vosgerau, G. (2010). Memory and content. Consciousness and Cognition, $19(3): 838-846$. 
Werning, M. and Cheng, S. (2017). Taxonomy and unity of memory. In Bernecker, S. and Michaelian, K., editors, The Routledge Handbook of Philosophy of Memory, pages 7-20. Routledge. 